\title{
Future Directions of Rheumatology Training According to Saudi Vision 2030: Recommendations From a Saudi Experts Meeting
}

This article was published in the following Dove Press journal:

Open Access Rheumatology: Research and Reviews

\author{
Rania Zaini ${ }^{1}$ \\ Ali Alrehaily ${ }^{2,3}$ \\ Ahmed Alhazmi ${ }^{4,5}$ \\ Hussein Halabi ${ }^{6}$ \\ Heba Mohamed $\mathbb{( D}^{7}$ \\ Hani Almoallim (iD) 8,9 \\ 'Department of Medical Education, \\ Faculty of Medicine, Umm Alqura \\ University, Makkah, Saudi Arabia; \\ ${ }^{2}$ Department of Medicine, Security \\ Forces Hospital Program, Riyadh, Saudi \\ Arabia; ${ }^{3}$ Chairman of the Scientific \\ Committee of Adult Rheumatology \\ Fellowship Program, Saudi Commission \\ for Health Specialties (SCFHS), Riyadh, \\ Saudi Arabia; ${ }^{4}$ Department of Medicine, \\ King Fahad Armed Forces Hospital \\ (KFAFH), Jeddah, Saudi Arabia; \\ ${ }^{5}$ Chairman, Supervisory Committee for \\ Saudi Board Adult Rheumatology \\ Fellowship Program in Western Region, \\ Saudi Commission For Health Specialties \\ (SCFHS), Riyadh, Saudi Arabia; \\ ${ }^{6}$ Department of Medicine, King Faisal \\ Specialist Hospital And Research Center, \\ Jeddah, Saudi Arabia; ${ }^{7}$ Pfizer \\ Biopharmaceutical Group, Emerging \\ Markets, Riyadh, Saudi Arabia; \\ ${ }^{8}$ Department of Medicine, Faculty of \\ Medicine, Umm Alqura University, \\ Makkah, Saudi Arabia; ${ }^{9}$ Alzaidi Chair of \\ Research in Rheumatic Diseases, Umm \\ Alqura University, Makkah, Saudi Arabia
}

Correspondence: Hani Almoallim 6604 Gais Bin Doraih, Alnaeem, Jeddah 23621-3514, Saudi Arabia

Email hmmoallim@uqu.edu.sa
Background: Worldwide research anticipates that a current shortage of rheumatologists will exacerbate over the next decade, whereas the need for arthritis specialists will continue to escalate. Saudi Arabia (SA) also encounters a limited geographical distribution of rheumatologists and rheumatology fellowship training centres.

Objective: Reporting the Saudi rheumatologists' advisory meeting conducted in Makkah, SA in January 2020 with the aim to discuss the "Saudi Vision 2030" for rheumatology training programs.

Materials and Methods: A meeting of Saudi rheumatology experts and consultants was conducted to address the future directions, challenges, and recommendations of rheumatology training. The 10th Rheumatology Practice Symposium was organised by Alzaidi Chair of Research in Rheumatic Diseases (ZCRD), and conducted in Makkah Commerce Chamber, Makkah, SA on January 28, 2020. More than 30 consultants and rheumatology fellows with five Saudi experts in the field of rheumatology assembled to form 10 recommendations that tackle rheumatology training challenges in SA.

Results: The meeting recommendations shed light on the clinical practice of rheumatology training in SA; challenges and opportunities in rheumatology fellowship programs; efforts of the Saudi Commission for Health Specialties (SCFHS) to design and implement a competent postgraduate rheumatology training; and challenges with trainers, trainee, and within training centres.

Conclusion: To address rheumatology challenges in SA, rheumatology consultants and fellows assembled to form 10 recommendations. The recommendations tackled the challenges of rheumatology fellowship programs and the efforts to implement a competent postgraduate rheumatology training. These recommendations are expected to lead us successfully to fulfil our ambition in the "Saudi Vision of 2030".

Keywords: rheumatology, residents, Saudi, fellowship, training, training program

\section{Introduction}

Rheumatology comprises the preventive, active, and rehabilitative treatment of patients who suffer from joint, musculoskeletal (MSK) and connective tissue diseases. ${ }^{1}$ Rheumatic diseases are chronic, debilitating, and progressive, and can lead to patients' disability. ${ }^{2}$ Disability from rheumatic disease has serious social and health implications, including limitations to patients' working performance and to their social functioning. Lumbalgia, for example, accounts for a considerable portion of economic losses due to disability and is estimated to affect over $60 \%$ of the 
Saudis (a percentage similar to other countries in Saudi Arabia's (SA's) demographic, but a far larger percentage than several Western countries like the United Kingdom or Canada). ${ }^{3}$ Almoallim et al have conducted a study on 197 Saudi patients to address the impact of rheumatoid arthritis (RA) on workability in a Saudi population and examined the correlation between objective measures of disease activity and reduced workability, highlighting that higher absenteeism and more activity impairment were seen for patients with persistent Disease Activity Score (DAS-28) $>3.2$ (non-achievers). ${ }^{4}$ Albers and colleagues investigated the socio-economic consequences in a three-year period of patients with RA and found that work disability is more profound in rheumatoid patients than in general population. $^{5}$

Studies show wide global discrepancy between the prevalence of rheumatic diseases and the availability of competent and qualified rheumatologists. ${ }^{6}$ Many countries, including SA, are facing a challenging shortage of rheumatologists, which affects many aspects of rheumatology medical care. ${ }^{5,7} \mathrm{~A}$ worldwide apprehension has also arisen that rheumatology has lost its appeal among internal medicine residents as a sub-specialty. ${ }^{2,5}$ Studies reported many challenges to the popularity of the speciality. Early exposure of internal medicine residents and students to rheumatology training is suggested to influence their sub-specialty decisions. ${ }^{7}$ The Saudi Commission for Health Specialties (SCFHS) adopted the CanMEDS framework ${ }^{8}$ to establish a core curriculum for all training programs including rheumatology. 9,10 Under the SCFHS rheumatology curriculum, Saudi physicians undergo two years of full-time supervised training in which they are actively involved in patient care, with responsibility increasing as further experience and competence are gained. ${ }^{10}$

The aim of this qualitative work is to explore the future directions of rheumatology training in SA. Our country is approaching a new era in its history outlined in the Saudi Vision 2030, an ambitious Saudi government initiative to diversify the national economy and to make public sector improvements in the areas of health, infrastructure, education, tourism, and other areas. Saudi Vision 2030 is expected to result in a massive overhaul of Saudi healthcare delivery, including reforms in the supervision of medical education. ${ }^{11}$

This innovative vision of development should be accompanied by clear strategies for training programs in different specialties. This manuscript is an initiative from the Alzaidi Chair of Research in Rheumatic Diseases (ZCRD) at Umm Alqura University and is produced in collaboration with SCFHS.

\section{Methods}

This is a novel qualitative study that is addressing challenges that have not been tackled before in the context of rheumatology. It is an opinion-based paper and collaborative view that serves as a future planning strategy that we are implementing to match the 2030 vision. The 10th Rheumatology Practice Symposium, organised by ZCRD, was conducted in Makkah Commerce Chamber in Makkah, SA on January $28,2020 .{ }^{12}$ A dedicated meeting to address the future directions, challenges, and recommendations of rheumatology training based on Saudi 2030 vision was arranged. This meeting aimed to discuss the clinical practice of rheumatology in SA and explore opportunities and challenges of rheumatology training programs to generate national recommendations to enhance the current rheumatology fellowship program and improve future practice. The research committee in ZCRD has approved this research protocol.

\section{Study Design}

The study utilised the nominal group technique (NGT), a validated method for group brainstorming and decisionmaking. It is considered "a structured meeting which seeks to provide an orderly procedure for obtaining qualitative information from target groups who are most closely associated with a problem area." 13 The NGT provided an encouraging environment for the group to identify, rank and rate critical problems in a relatively egalitarian fashion.

\section{Study Setting}

The study supplemented the 10th Rheumatology Practice Symposium, which took place on January 28, 2020. The study consisted of 35 participants (30 rheumatology consultants and fellows, plus five Saudi rheumatology expert consultants). The meeting panel consisted of these five experts in the field of rheumatology from various institutes in SA and attended by more than 30 consultants and fellows. The experts consist of:

- The chair of rheumatology fellowship training programs in Saudi Arabia.

- The chair of the Western committee of rheumatology fellowship training programs. 
- The director of academic and training affairs in King Faisal Specialist Hospitals in Jeddah, Saudi Arabia, who is also a former president of the Saudi Society of Rheumatology.

- The Alzaidi Chair of Research in Rheumatic Diseases.

- A national expert in medical education who chairs the Department of Medical Education at the Medical College of Umm Alqura University in Makkah, Saudi Arabia.

The experts, consultants, and fellows comprise a diverse geographical sampling across SA's population, with rheumatologists representing the Western, Middle, Eastern, Southern, Asir, and Madina regions all present. The first four listed experts are rheumatologists by training, all with at least 18 years of experience training residents and fellows. The head of the scientific committee of the adult rheumatology fellowship program in SA is one of the experts and authors of this collaborative work. The meeting addressed: (1) exploring the challenges of local rheumatology practice in accordance with the vision of 2030; (2) exploring opportunities and challenges of rheumatology training programs; (3) discussing the shared responsibilities of all related parties; and (4) the quality measures of training programs and activities. All recommendations from this meeting were unanimously agreed upon by the panel and were collected in this paper. The meeting was reported according to the standards for reporting qualitative research (SRQR).

\section{Data Collection}

The aim of the study and meeting was presented, and essential published facts were highlighted to all participants. Afterward, participants were asked to outline the top challenges of rheumatology fellowship programs in SA and suggested solutions. Then, the panel (five experts) and attended (30 rheumatology fellows and consultants) shared their ideas and listed the suggested challenges and solutions. Subsequently, the group voted for the listed challenges and solutions to prioritise the top ten. Finally, the generated consensus was discussed among the group members.

\section{Results}

The panel and group outlined challenges of rheumatology fellowship programs in SA and suggested empowering solutions. ${ }^{14,15}$ The vote and rank of the suggested main challenges and solutions developed a consensus of seven challenges (41\% agreed that these were the most important challenges to consider) and ten main recommendations ( $48 \%$ agreed on these ten recommendations), which were clustered to present a comprehensive view of the discussion. Table 1.

During the first open meeting attended by all participants (35 participants and led by expert No. 5), all inquiries, challenges, and concerns were addressed and recorded. Based on the discussions that were organically generated, 10 recommendations emerged. Following the meeting, a consensus was attained among the five experts on all of these recommendations. Expert 5 wrote the recommendations, and these were shared to all authors who added to, adjusted to, or modified in three rounds all these recommendations, until all experts came to an agreement about all of them.

The suggested recommendations address the goals of Saudi vision 2030 in relation to increasing the efficiency of the health sector and enhancing capabilities of national

\section{Table I Primary Challenges}

\section{Primary Challenges:}

The panel discussed the 2030 vision of SA that was addressed by the SCFHS effort to improve the residency and fellowship training programs as part of a competent, efficient health care system. The Commission adopted the Canadian competency framework ${ }^{8}$ (CanMEDS) and developed the training requirements, and then introduced the work-place assessment initiatives. ${ }^{10}$ Yet some of the challenges were addressed:

I. The shortage of rheumatologists, a global concern especially with the growing number of patients with arthritis and other rheumatic conditions worldwide.

2. The limited geographical distribution of rheumatologists and rheumatology fellowship programs in SA.

3. The lack of basic core MSK clinical competencies amongst general practitioners and clinical specialists, which has a serious impact on patient outcomes.

4. The absence of national standardised referral criteria and MSK clinical practice guidelines.

5. Limited training opportunities for fellows and poor mentoring and feedback.

6. The discrepancy between training centres in the quality of delivered training.

7. Consultants and fellows' inadequate core clinical skills especially with the reliance on technology in investigation. 


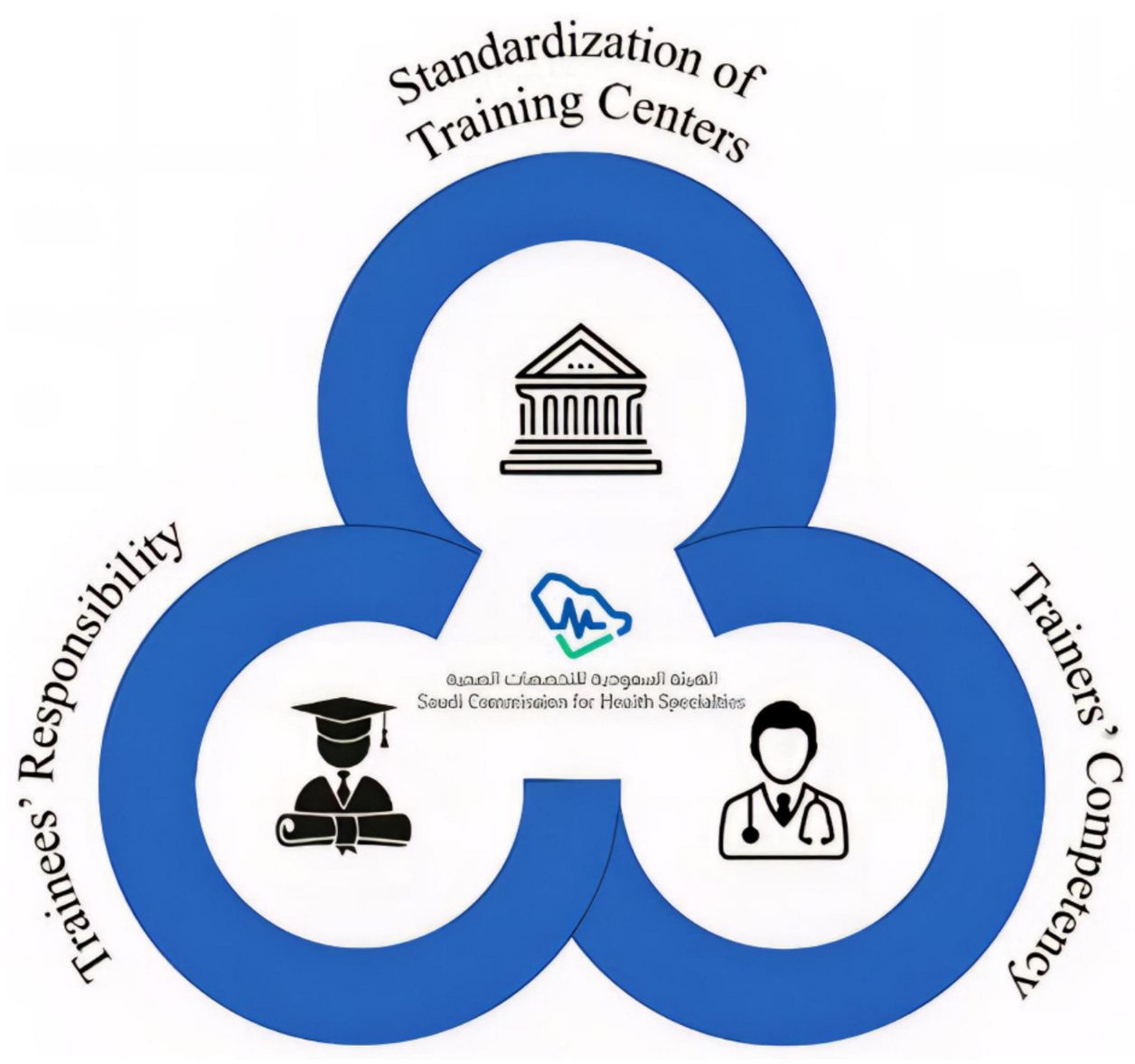

Figure I Competency triangle for rheumatology training program: Saudi 2030 Vision.

staffs. The recommendation advocates a "triangle" of competency for the rheumatology training program per the Saudi Vision 2030 (Figure 1) in four interrelated main domains: 1) standardisation of the training centre; 2) developing trainers' competency; 3) emphasising fellows and trainees' responsibilities; 4) developing a shared governance between the SFCHS and training parties.

Since these recommendations do not attempt to manage a certain disease, and nor do they represent an articulation of a randomised controlled trial, the recommendations were not graded. All of these recommendations are important for the future directions for rheumatology training. Some of them may be less applicable in some centres as compared to other centres.

\section{Recommendation I}

Develop an MSK core capabilities framework and emphasise suitable training exposures to general practitioners and residents. The core competencies must be addressed in all undergraduate and relevant residency programs. Integrating the rheumatology competencies in undergraduate and some residency programs will equip general practitioners and clinical specialists with the required competencies to improve the provided services and ensure early diagnosis, prevention and management of MSK conditions. Also, the right comprehensive exposure to rheumatology in undergraduate and related general residency programs will recruit more interested, competent candidates to the specialty.

\section{Recommendation 2}

Develop standardised and validated criteria for referral to a rheumatologist. The standardised and validated criteria will help primary care physicians and other specialties to identify individuals who need referral to a rheumatologist. Implementing the criteria will facilitate the early diagnosis and initiation of treatment improving patients' outcomes.

\section{Recommendation 3}

Develop standardised investigation protocols and clinical examinations for trainees and emphasise the core clinical skills of fellows. The mass utilisation of technology in clinical investigations is indicative of a schism between 
current practice and basic training. Currently, many rheumatologists scantily perform physical examinations to develop a diagnosis, overly relying on X-rays and images. Yet physical examination is a core skill for rheumatology and must be gained and assessed.

\section{Recommendation 4}

Increase the number of trainees and training centres, especially in the underserved cities; introduce elective rotations in community-based rheumatology teaching clinics, underserved centres, and rural areas as part of the fellowship core training.

\section{Recommendation 5}

Ensure comprehensive training that covers the essential competencies of CanMEDS. ${ }^{8}$ Implement the fellowship training activities to focus not only on clinical competencies but on other core competencies as scholarship, professionalism, and health advocacy. Structured journal clubs, evidence-based medicine, and research activities should be essential parts of the training program.

\section{Recommendation 6}

Ensure trainers and trainees' ownership of the program and its development plans. The SCFHS efforts to develop and standardise the trainings are well recognised. Yet sharing their plans with stakeholders is urgent. Trainers and trainees sometimes disagree with or misunderstand the suggested development plans. This is an understandable reaction to any changing initiatives. Nevertheless, sharing plans and providing good training on these plans will facilitate a shared governance.

\section{Recommendation 7}

Standardise the training centres. Each training centre must provide a suitable training environment. Hospital policies and resources must be reviewed to support residents and fellows. Empowering trainees is an essential aspect in clinical facilities that couple clinical practice with lifelong learning.

\section{Recommendation 8}

Prioritise trainer competency. Ensure adequate training of the trainers. All consultants and trainers must be trained to teach and assess residents and fellows. Providing frequent evaluation and feedback for fellows and residents will ensure their growth and readiness.

\section{Recommendation 9}

Develop the training programs with workplace-based learning and assessment, which empower fellows as directed trainees. Program directors and trainers must design relevant workplace-based teaching and assessment approaches that are supported with immediate and constructive feedback. Introduce peer-assisted teaching, through which senior fellows teach and assess juniors.

\section{Recommendation 10}

Merge clinical practice, training, and research. Create means to encourage health facilities, consultants, and trainees to develop different clinical care projects. Based on the diversity of rheumatology that intermingles with different specialties, these projects can be in a form of combined services with other specialists like dermatology, orthopaedics, ophthalmology, obstetrics, or gynaecology. Alternatively, a highly specialised clinic in one rheumatological disease such as systemic lupus erythematosus (SLE) can be developed to dedicate clinical care and research to patients with this disease. Local registries for diseases like RA should also be encouraged.

Additionally, the panel advised that all rheumatologists should meet the minimum requirement of competence to perform high-quality and safe patient care, which was also advised by the 2019 EULAR recommendations ${ }^{16}$ for the generic core competencies of health professionals in rheumatology addressed in the SCFHS guidelines.

\section{Discussion}

The developed recommendations from the Saudi experts meeting aim to address the future directions of training in rheumatology according to Saudi Vision 2030. ${ }^{17}$ The vision aims to increase the efficiency of the health sector and enhance the capabilities of national staff.

Literature suggests that the lack of official information about and lack of direct exposure to rheumatology during undergraduate studies or general residency programs contribute to a shortage of trainees in rheumatology. ${ }^{14,18-22}$ A questionnaire-based study by Zaini et al on 296 residents of internal medicine in SA showed that residents expressed low self-confidence in demonstrating MSK physical examinations, with a total mean of (10/24) suggesting inadequate MSK education, during and post, medical school training. ${ }^{20}$ A quasi-experimental study conducted by Magliah et al in Jeddah, SA evaluated the agreement between rheumatologists and primary care physicians (PCPs) in detecting 
inflammatory arthritis (IA) when applying the standardised MSK examination techniques, highlighting that a refinement of the standardised training process could further improve accuracy and help PCPs to confidently identify cases of early IA. ${ }^{15}$ In efforts to standardise MSK examination of the hand and wrist joints, Almoallim et al clinically assessed a total of 2112 joints using MSK examination and ultrasound (US), showing that MSK examination has $69-80 \%$ sensitivity when compared with the US. ${ }^{23}$ Therefore, the implementation of early integration of rheumatology curriculum during undergraduate years and residency training programs becomes a necessity. ${ }^{20,21}$ In addition, this early integration should be based on clear emphasis on standardised MSK examination techniques.

Rheumatology in undergraduate education in SA is taught mostly as part of the internal medicine curriculum with minimum exposure to clinical settings. In this regard, Umm Alqura University launched a reformed MBBS curriculum in $2016 .{ }^{24}$ The curriculum introduced two spiral musculoskeletal modules in Year 3 and Year 6 of the sixyear program. The courses integrated rheumatology with orthopaedics within related underpinning clinical sciences to address MSK disorders and wellbeing, with direct exposure in primary health-care centres, out-patients clinic, inpatient ward, emergency rooms, and physiotherapy.

The recommendations to develop standardised and validated referral criteria based on standardised MSK examination and investigation protocols facilitate the development of a continuum and clarity about the healthcare system, which has positive economic implications. Moreover, the recommendations implore collaboration between the Ministry of Health (MOH) and SCFHS to standardise the referral system for convenient access to rheumatology facilities. A Saudi study by ZCRD in collaboration with $\mathrm{MOH}$ was conducted to validate the parameters that should be used in a referral criteria. ${ }^{24}$ There was a total of nine variables identified as having high specificity and good predictive value: loss of appetite, swelling of metacarpophalangeal joint 2 or 5, swelling of proximal interphalangeal joint 2 or 3, wrist swelling, wrist tenderness, a positive test for rheumatoid factor, and a positive test for anti-citrullinated protein antibodies. ${ }^{24}$ This work needs to progress to validate a core set of a criteria used for referral. Many other studies confirmed that the communication between PCP and rheumatologists is fundamental to appropriately and promptly diagnose and treat rheumatic patients and ensure the continuity of care. $^{25-27}$
Much of the promise that these recommendations hold for improving rheumatology education and collaboration between trainees and practitioners is threatened by the COVID-19 pandemic. Like other specialties, rheumatology is learning to adapt the delivery of education and training content in the wake of new COVID-19-mandated regulations. A nationwide cross-sectional study of residents and fellows in several specialties in SA reported that $84.6 \%$ of the respondents reported a reduction in training activities due to the COVID-19 pandemic. ${ }^{28}$ The authors of the study recommended the use of e-learning platforms to continue some educational activities. ${ }^{28}$

The presence of COVID-19 has accelerated the creative usage of digital communication and dissemination of educational materials. Social media channels have become instrumental to the future of rheumatology education, with online journal clubs, more opportunities for interactive educational formats, and other benefits. ${ }^{29}$ Though this study was conducted before the global spread of COVID-19, the pandemic has changed the fundamental delivery of rheumatology education, perhaps irrevocably.

Other challenges include the unbalanced geographical distribution of service and the shortage of the rheumatology workforce. ${ }^{30}$ A retrospective study conducted by Hussain et al on 250 RA patients from six hospitals in SA concluded that geographic distribution influenced RA diagnosis, with rural patients experiencing a greater delay than urban patients $(\mathrm{p}<0.0001) .{ }^{31}$ Thus, it is recommended to increase the number of trainees and training centres especially in the underserved cities and introduce elective rotations in community-based rheumatology clinics, underserved medical centres, and rural areas as part of the rheumatologist core training.

The panel's minimum requirement recommendation for rheumatologists highlighted the necessity of ensuring comprehensive training that covers the essential competencies of CanMEDS. ${ }^{8}$ Successful training experiences require a clear competencies-based education framework, engaging learning opportunities and structure assessment and feedback. Nevertheless, some training centres only addressed the clinical competencies and overlooked other competencies of rheumatologists, such as performing research and applying research evidence in practice. Other centres only focused on the practice and provided no or minimal opportunity for training and follow-up of trainees' progress. That might be due to SCFHS guidelines and development plans being overlooked amongst trainers and trainees, or the training centres placing inadequate 
priority on fellows' learning. The panel addressed such challenges and recommended that ownership of the SCFHS development plan among trainees and trainers be maximised. The $\mathrm{MOH}$ or other juristic bodies should also place priorities into clinical training by merging clinical practice and training.

The SCFHS ${ }^{9,10}$ invests comprehensively in developing residency and fellowship programs, by adopting the CanMEDS $^{8}$ competencies framework for all training programs and developing training and assessment practises. Around the country, approximately 34 trainees are currently enrolled in a rheumatology training program and are distributed throughout the accredited training centres in the central, eastern, southern, and western region of the kingdom. Research has become mandatory in some specialities, including rheumatology. Each fellow is obligated to complete the assigned research projects within two years and publish at least one scientific paper in a peer-reviewed journal. ${ }^{16}$ In addition, workplace-based assessment was adopted as a philosophy. Each fellow is also required to complete a set of clinical tasks under supervision and get feedback which include mini-clinical evaluation exercise (Mini-CEX), ${ }^{32}$ direct observation of procedure skills (DOPS), case-based discussion, cognitive and attitude skills, specific academic activity, and procedure logbook. Therefore, SCFHS efforts must be combined with 1) developing trainers and trainees' ownership of the addressed changes to ensure better implementation; 2) intensive training for trainers for proper training, assessment, and feedback for trainees; and 3) protecting trainees' time as recommended in this study.

The discrepancy between a theoretical curriculum and current clinical practice is one of the main challenges of implementing the training program. Applying a standardisation of the medical training among all centres and creating more fellowship programs for subspecialties within the rheumatology field, such as osteoarthritis and musculoskeletal ultrasound training, ${ }^{33,34}$ ensure a smooth flow of the training fellowship and globally improves training quality.

Miscommunication between fellows and directors of training centres could represent a further challenge to the training program. Hence, designing an internal policy to support the fellows throughout training, evaluating the program directors, sending the evaluation forms to the Saudi Commission periodically, and informing the trainees of their rights are recommended to enhance the communication between the program directors and the trainees.

To create stronger programs, rheumatology educators may also need to develop new competencies.
Consequently, it is recommended to provide all trainers and mentors with Training of the Trainers (TOT) courses to ensure the adequacy of the rheumatology education. These recommendations can help reframe rheumatology training programs among residents and fellows by providing an integrated MSK exam curriculum, including standardised teaching of clearly defined MSK examination techniques and skills.

A proposed recommendation by the panel was to combine rheumatology and other specialty clinics care through a dual-treatment clinic. The suggestion follows an ongoing trend by academic medical centres to integrate specialties that treat overlapping conditions. The clinical and research benefits of this collaboration have been previously described to contribute to a better and more sustainable health-care system. ${ }^{35}$ The panel has also recommended the implementation of an outpatient disease specialised clinic to provide better health-care privileges for patients affected by one disease like RA or SLE. The idea was studied by Rubio et al in Spain to assess the clinical practice in a clinic model specialising in chronic obstructive pulmonary disease (COPD). They emphasised that the COPD patients who were treated in a specialised clinic showed greater clinical control of COPD compared to those treated at conventional clinics, despite the specialised clinic seeing patients with more advanced disease. ${ }^{36}$ The panel also encouraged the implementation of local registries for RA. The registries can present information on several aspects including clinical guidelines and quality of care, drug safety and benefit-risk data, and healthrelated outcomes, including quality of life and clinical effectiveness. ${ }^{37}$ There are successful examples of local efforts in building registries in SA like the efforts conducted by ZCRD to build the Rheumatoid Arthritis Saudi Database (RASD). ${ }^{4}$ However, collaboration of efforts is greatly needed to expand the impact of these initiatives.

This qualitative study has several limitations. Accurate replication of the findings obtained here might not be a feasible feature. Reliability is always a concern with qualitative research methodology. This work is based on the opinion of the participants and the discussion created around the issues that were addressed. Compared to the Delphi technique, the nominal group technique used to generate participation is highly controlled and targeted for specific discussion, which can limit free idea exchange and development. The aim was to study our complex reality and examine factors affecting it to deepen our understanding. Challenges that were 
identified and subsequently the recommendations that were developed obviously are not statistically representative. It might be a feasible option to obtain quantitative agreement on our findings by wider dissemination to a larger group of experts and stakeholders. In addition, our findings are time-sensitive.

\section{Conclusion}

The need for rheumatology specialists is accelerating worldwide. SA is challenged by a limited geographical distribution of rheumatologists and rheumatology fellowship programs. This Saudi rheumatologists' advisory meeting made a concerted effort to provide recommendations to achieve the Saudi Vision 2030 for rheumatology training programs. The meeting yielded ten recommendations that tackle all persisting challenges through developing MSK core capabilities framework, increasing the number of trainees and training centres in the underserved cities, standardising the training centres, and merging rheumatology clinical practice with training and research. Implementation of these recommendations has the potential to increase the reach, quality, and sustainability of current Saudi rheumatology training.

\section{Author Contributions}

All authors contributed to data analysis, drafting or revising the article, have agreed on the journal to which the article will be submitted, gave final approval of the version to be published, and agree to be accountable for all aspects of the work.

\section{Disclosure}

Prof. Dr. Hani Almoallim report: This work was supervised and partially funded by Alzaidi Chair of research in rheumatic diseases. It was also partially funded by Pfizer pharmaceuticals. The authors report no other conflicts of interest in this work.

\section{References}

1. Pereira da Silva JA, Woolf AD, da Silva JAP, Woolf AD. The Importance of Rheumatic Diseases. In: Da Silva JAP, Woolf AD, editors. Rheumatology in Practice. London: Springer; 2010:1-13.

2. Kłak A, Raciborski F, Samel-Kowalik P. Social implications of rheumatic diseases. Reumatologia. 2016;2:73-78. doi:10.5114/reum.2016. 60216

3. Aldera MA, Alexander CM, McGregor AH. Prevalence and incidence of low back pain in the kingdom of saudi arabia: a systematic review. J Epidemiol Global Health. 2020;10:269-275.
4. Almoallim H, Janoudi N, Alokaily F, et al. Achieving comprehensive remission or low disease activity in rheumatoid patients and its impact on workability - saudi rheumatoid arthritis registry. Open Access Rheumatol Res Rev. 2019;11:89-95.

5. Albers JM, Kuper HH, van Riel PL, et al. Socio-economic consequences of rheumatoid arthritis in the first years of the disease. Rheumatology (Oxford). 1999;38(5):423-430. doi:10.1093/rheumatology/38.5.423

6. Al Maini M, Adelowo F, Al Saleh J, et al. The global challenges and opportunities in the practice of rheumatology: white paper by the world forum on rheumatic and musculoskeletal diseases. Clin Rheumatol. 2015;34(5):819-829. doi:10.1007/s10067-0142841-6

7. Kolasinski SL, Bass AR, Kane-Wanger GF, et al. Subspecialty choice: why did you become a rheumatologist? Arthritis Care Res. 2007;57(8):1546-1551. doi:10.1002/art.23100

8. Frank JR, Snell L, Sherbino J, editors. CanMEDS 2015 Physician Competency Framework. Ottawa: Royal College of Physicians and Surgeons of Canada; 2015. Accessed on June 11.

9. Saudi Commission For Health Specialties. Saudi board internal medicine curriculum. Available from: https://www.scfhs.org.sa/MESPS/ TrainingProgs/TrainingProgsStatement/Documents/INTERNAL\% 20MEDICINE.pdf. Accessed June 11, 2020.

10. Saudi Commission For Health Specialties. Saudi rheumatology fellowship curriculum; 2019. Available from: https://www.scfhs. org.sa/MESPS/TrainingProgs/TrainingProgsStatement/Documents/ SAUDI_RHEUMATOLOGY_FELLOWSHIP.pdf. Accessed June 11,2020 .

11. Full text of Saudi Arabia's Vision 2030. english.alarabiya.net. Accessed November 162020.

12. The 10th rheumatology practice symposium. Yahia and Mashaal AlZaidi, Sons of Sheikh Sorour Al-Zaidi, Chair of Research in Rheumatic Diseases - Deanship of Scientific Research | Umm Al-Qura University. Available from: https://uqu.edu.sa/en/App/Events/13768.

13. Ven A, Delbecq A. The nominal group as a research instrument for exploratory health studies. Am J Public Health. 1972;62(3):337-342. doi:10.2105/AJPH.62.3.337

14. Renner BR, DeVellis BM, Ennett ST,et al. Clinical rheumatology training of primary care physicians: the resident perspective PubMed. J Rheumatol. 1990;17:666-672.

15. Magliah R, Hafiz W, Alahmadi ZA, et al. Early diagnosis of inflammatory arthritis by primary care physicians following training by a rheumatologist. Open Access Rheumatol Res Rev. 2019;11:315-321.

16. Edelaar L, Nikiphorou E, Fragoulis GE, et al. 2019 EULAR recommendations for the generic core competencies of health professionals in rheumatology. Ann Rheum Dis. 2020;79(1):53-60. doi:10.1136/ annrheumdis-2019-215803

17. Saudi Vision 2030. Available from: https://vision2030.gov.sa/sites/ default/files/report/Saudi_Vision2030_EN_2017.pdf. Accessed on June 112020.

18. Lim SY, Bolster MB. Challenges in optimizing medical education for rheumatologists. Rheum Dis Clin North Am. 2019;45(1):127-144. doi:10.1016/j.rdc.2018.09.008

19. Al Maini M, Al Weshahi Y, Foster HE, et al. A global perspective on the challenges and opportunities in learning about rheumatic and musculoskeletal diseases in undergraduate medical education: white paper by the World Forum on Rheumatic and Musculoskeletal Diseases (WFRMD). Clin Rheumatol. 2020;39(3):627-642. doi:10. 1007/s10067-019-04544-y

20. Zaini R, Almoallim H, Hafiz W, et al. Musculoskeletal teaching and training in saudi internal medicine residency programmes. Creat Educ. 2016;07(06):824-830. doi:10.4236/ce.2016.76085

21. Almoallim H, Gelidan A. Musculoskeletal examination skills: are we still interested? Int J Clin Med. 2012;03(05):335-340. doi:10.4236/ ijcm.2012.35065 
22. Almoallim H, Khojah E, Allehebi R, Noorwali A. Delayed diagnosis of systemic lupus erythematosus due to lack of competency skills in musculoskeletal examination. Clin. Rheumatol. 2007;26(1):131-133. doi:10.1007/s10067-006-0225-2

23. Almoallim H, Attar S, Jannoudi N, et al. Sensitivity of standardised musculoskeletal examination of the hand and wrist joints in detecting arthritis in comparison to ultrasound findings in patients attending rheumatology clinics. Clin. Rheumatol. 2012;31(9):1309-1317. doi:10.1007/ s10067-012-2013-5

24. Faculty of Medicine. Umm Al-Qura University. Available from: https://uqu.edu.sa/colmedsc. Accessed June 25, 2020.

25. Almoallim H, Janoudi N, Attar SM, et al. Determining early referral criteria for patients with suspected inflammatory arthritis presenting to primary care physicians: a cross-sectional study. Open Access Rheumatol Res Rev. 2017;9:81-90.

26. Wong J, Tu K, Bernatsky S, et al. Quality and continuity of information between primary care physicians and rheumatologists. $B M C$ Rheumatol. 2019;3(1). doi:10.1186/s41927-019-0067-6.

27. Fitzgerald A, de Coster C, McMillan S, et al. Relative urgency for referral from primary care to rheumatologists: the priority referral score. Arthritis Care Res. 2011;63(2):231-239. doi:10.1002/acr.20366

28. Balhareth A, AlDuhileb MA, Aldulaijan FA, Aldossary MY. Impact of COVID-19 pandemic on residency and fellowship training programs in Saudi Arabia: a nationwide cross-sectional study. Ann Med Surg. 2020;57:127-132. doi:10.1016/j.amsu.2020.07.025

29. Ahmed S, Zimba O, Gasparyan AY. Moving towards online rheumatology education in the era of COVID-19. Clin Rheumatol. 2020;39 (11):3215-3222. doi:10.1007/s10067-020-05405-9

30. Block SL, Hedrick J, Tyler R, et al. Increasing bacterial resistance in pediatric acute conjunctivitis (1997-1998). Antimicrob Agents Chemother. 2000;44(6):1650-1654. doi:10.1128/AAC.44.6.16501654.2000
31. Hussain W, Noorwali A, Janoudi N, et al. From symptoms to diagnosis: an observational study of the journey of rheumatoid arthritis patients in Saudi Arabia. Oman Med J. 2016;31(1):29-34 doi:10.5001/omj.2016.06

32. American Board of Internal Medicine. Mini-CEX. https://www.abim. org/program-directors-administrators/assessment-tools/mini-cex. aspx. Accessed June 11, 2020.

33. Battistone MJ, Barker AM, Grotzke MP, et al. "Mini-Residency" in musculoskeletal care: a national continuing professional development program for primary care providers. J Gen Intern Med. 2016;31 (11):1301-1307. doi:10.1007/s11606-016-3773-4

34. Miloslavsky EM, Criscione-Schreiber LG, Jonas BL, et al. Fellow as teacher curriculum: improving rheumatology fellows' teaching skills during inpatient consultation. Arthritis Care Res. 2016;68 (6):877-881. doi:10.1002/acr.22733

35. Theodorakopoulou E, Dalamaga M, Katsimbri P, Boumpas DT, Papadavid E. How does the joint dermatology-rheumatology clinic benefit both patients and dermatologists? Dermatol Ther. 2020;33 (3):13283. doi:10.1111/dth.13283

36. Rubio MC, Soler-Cataluña JJ, López-Campos JL, et al. Assessing the clinical practice in specialised outpatient clinics for chronic obstructive pulmonary disease: analysis of the EPOCONSUL clinical audit. PLoS One. 2019;14(2)::e0211732

37. Trotter JP. Patient registries: a new gold standard for "real world" research. Ochsner J. 2002;4:211-214.
Open Access Rheumatology: Research and Reviews

\section{Publish your work in this journal}

Open Access Rheumatology Research and Reviews is an international, peer-reviewed, open access journal publishing original research, reports, editorials, reviews and commentaries on all aspects of clinical and experimental rheumatology in the clinic and laboratory including the following topics: Pathology, pathophysiology of rheumatological diseases; Investigation, treatment and management

\section{Dovepress}

of rheumatological diseases; Clinical trials and novel pharmacological approaches for the treatment of rheumatological disorders. The manuscript management system is completely online and includes a very quick and fair peer-review system, which is all easy to use. Visit http://www.dovepress.com/testimonials.php to read real quotes from published authors. 